\title{
La indefensión de los filósofos ante el desafío moral de nuestro tiempo*
}

\author{
ERNST TUGENDHAT \\ Universidad Libre de Berlín
}

A la hora de encarar los problemas morales de nuestro tiempo, cabría hacerlo desde dos perspectivas bien distintas. $\mathrm{La}$ primera se atendría a la fundamentación de la moral en general, mientras que la segunda se preocuparía por su extensión, interesándole averiguar quiénes son exactamente aquéllos para con los cuales nos vemos obligados moralmente. El primer aspecto es una consecuencia del proyecto ilustrado de procurar a la ética una fundamentación racional que viniese a sustituir a la religioso-tradicional. Las corrientes utilitarista y contractualista son tomadas como puntos de referencia para este análisis. La segunda perspectiva aborda una serie de problemas hacia los que sólo nos hemos sensibilizado desde hace unas pocas décadas. En definitiva se trata de responder a la pregunta de quién compone la comunidad ética. ¿Acaso contamos realmente con las generaciones venideras, los disminuidos físicos y psíquicos, o los propios niños, cuando compulsamos nuestras obligaciones morales?

Podría hablar de la indefensión de todos nosotros, en lugar de referirme únicamente a la de los filósofos. Todos nosotros nos enfrentamos hoy día con cuestiones morales fundamentales, que no podemos dejar de contestar de una manera $u$ otra, aunque no encontremos ninguna respuesta que nos satisfaga. Se trata de cuestiones filosóficas, dado que no podemos esperar de determinadas disciplinas científicas ninguna respuesta relativa a las mismas; son cuestiones que sólo pueden dilucidarse en una reflexión general, careciendo de importancia si dicha reflexión se califica o no como filosófica.

A decir verdad, la reflexión moderna acerca de la moral ha venido mostrándose bastante infructuosa, pero la consecuencia que muchos han extraído de ello, esto es, que la moral debe ser considerada como algo meramente subjetivo, tampoco representa una solución, ya que un subjetivismo semejante constituye tan sólo una actitud verbal; en la vida real emitimos constantemente juicios morales objetivos: juzgamos, por ejemplo, si se practica la tortura o si alguien no mantiene una promesa, sin

* Versión castellana de Concha Roldán Panadero. 
expresar con ello un sentimiento subjetivo, sino exigiendo de los demás un comportamiento recíproco. Si fuéramos realmente subjetivistas, tendríamos que cambiar todo nuestro comportamiento intersubjetivo de un modo que cuesta imaginar. La única forma adecuada de reaccionar ante la infructuosidad de la reflexión filosófico-moral llevada a cabo hasta el momento es contraponer a las dificultades soluciones sencillas, dando cuenta de ellas lo más concretamente posible.

Quisiera ocuparme a continuación de las dificultades morales en dos niveles bien diferenciados. El primero atañe a la fundamentación moral en general. El segundo a su extensión, es decir, a la cuestión de saber quiénes son aquellos para con los que tenemos deberes morales. El primer aspecto nos preocupa desde los siglos XVII y XVIII, lo mismo que habia preocupado ya a los griegos de los siglos $\mathrm{V}$ y $\mathrm{IV}$ a.C. Es el reto específico de la Ilustración: que la moral deje de justificarse por la tradición y las creencias religiosas, y comience a exigir algún tipo de fundamentación natural. Se han hecho diferentes intentos para conseguir dicha fundamentación natural. Pero la profunda indefensión en la que nos encontramos respecto a la moral da fe de que ninguno de esos intentos resulta tan convincente como para recibir la aprobación general. Cuando en el título de mi trabajo hablo de los desafíos morales de nuestro tiempo, me refiero al período de este primer nivel, en el cual nos encontramos desde hace al menos doscientos años.

El segundo nivel, en cambio, cl que se refiere a la cuestión de averiguar para con quién tencmos deberes morales, abarca una serie de problemas que sólo en las últimas décadas se nos han presentado en toda su magnitud. Hasta casi mediado nuestro siglo era usual representarse la comunidad moral como constituida mediante deberes recíprocos entre personas adultas vivas $\mathrm{y}$, aunque se pretendiera contestar teóricamente de forma afirmativa a la pregunta de si con ello se hacía referencia a todas las personas, lo cierto es que en la ética individualista se pensaba ante todo en el entorno inmediato y en la ética política en una configuración estatal muy determinada.

Sólo después de la segunda guerra mundial se ha ampliado la conciencia de que nuestra responsabilidad moral se extiende a todos los hombres. Pero esta conciencia no se mantiene de forma indiscutible, según se pone de manifiesto en cuestiones tales como la inmigración y el asilo político, o en qué medida la depauperación en el tercer mundo es un problema moral que nos atañe; se enfrentan aquí una conciencia moral más universalista y una más etnocéntrica. La universalista insiste en que la comunidad moral se compone de todos los seres humanos.

Ahora bien, este planteamiento acaba por conducirnos a una pregunta previa. Pues, ¿qué significa «todos los hombres»? Hasta hace poco 
hemos dado por supuesto que se hace referencia a todos los seres humanos que existen actualmente. Los problemas ecológicos, los cuales pueden tener consecuencias en parte irreversibles para las generaciones futuras, nos han llevado a analizar la cuestión acerca de si no tendremos también una responsabilidad moral para con esas generaciones venideras.

Pero ésta no es la única dimensión que se ha vuelto problemática en la pregunta "¿quiénes son todos?". Menciono de forma meramente marginal los problemas referentes al racismo y al sexismo, porque a pesar de constituir serios problemas prácticos, apenas entrañan ambigüedad alguna de cara a la reflexión moral teórica. Topamos con dificultades más complejas en lo que respecta a los disminuidos físicos y psíquicos. Estos pueden representar un problema específico para determinadas teorías morales en tanto que no hay lugar en esos casos para la reciprocidad, pudiendo volverse unívoca la obligación moral, tal y como era el caso en lo referente a las generaciones futuras. No obstante, nos enfrentamos con una dificultad mucho mayor si se suprimen las funciones psiquicas, bien totalmente, bien hasta el punto en que se tenga que decir: ciertamente son seres humanos, pero no personas. Como criterio para la personalidad, no sólo se toma en consideración la racionalidad, sino especialmente la autonomía, la capacidad de determinar uno mismo el cómo de la propia vida. Está implícito el que esos seres humanos no son por su parte sujetos morales, de forma que topamos aqui con la cuestion siguiente, a saber, en qué medida seres que no son sujetos morales pueden ser por su parte objetos de obligación moral.

Un problema semejante se nos presenta en lo que respecta a embriones, fetos y niños pequeños. En este punto se plantea frecuentemente la cuestión: "¿En qué momento comienza un embrión a ser un ser humano?». Ahora bien, si la característica decisiva que tenemos presente, cuando hablamos de los seres humanos en un sentido moral, fuera de la «ser una persona», el recién nacido es sin duda un ser humano, mas todavía no alcanza la categoría de persona. Y si nos preguntamos por una característica que sea moralmente relevante en el tiempo, previa al hecho de que un ser humano sea considerado como persona, ésta parece consistir en que se trata de un ser sensible, especificamente capaz de sentir dolor. Pero también los animales son seres sensibles. Por consiguiente, si se muestra la sensibilidad como caracteristica decisiva de la responsabilidad moral para con un objeto, entonces ya no podemos continuar restringiendo al concepto de ser humano la cuestión del alcance de la responsabilidad moral. Un ser humano, esto es, perteneciente a la especie biológica homo sapiens, es desde un principio un embrion humano, pero en las primeras semanas carece de conciencia, por lo tanto, tampoco tiene sensibilidad alguna. Así podría resultar que existiesen se- 
res humanos hacia los que no tuviéramos responsabilidad moral en absoluto, y que, por otra parte, existiesen otros seres hacia los que tuviéramos responsabilidad moral. Parece, pues, que los conceptos moralmente relevantes son, por una parte, el de ser persona y, por otra, la capacidad de sentir. Así pues, si se vuelve a plantear de forma más precisa la cuestión de hasta dónde se extiende la responsabilidad moral, el concepto de ser humano parece perder el carácter de guía que parecía tener en un primer momento, cuando era lógico preguntar: sí, todos los seres humanos, pero ¿quiénes son todos los seres humanos? ¿Cómo se explica que el concepto de ser humano pudiera parecernos tan central, aunque no entrañase para nada criterio alguno de diferenciación conceptual aparte de la pertenencia a una especie biológica? Al menos, encontramos un argumento de peso a su favor en nuestra tradición judeo-cristiano-islámica. Según la Biblia, Dios ha creado al hombre a su imagen y semejanza. De esto resulta, para la moral judeo-cristiana-islámica, que toda vida humana, si bien sólo ella, es sagrada. Todos nosotros nos hallamos todavía más o menos inmersos en esta tradición y por eso resulta intuitivamente obvia para la mayoría de nosotros esa concepción moral básica. Sin embargo, existen otras tradiciones religiosas con otras concepciones. Y tan pronto como reflexionamos espontáneamente, sin basarnos simplemente en las historias bíblicas, el concepto de ser humano pierde ese claro contorno que le confirió el estatus de línea divisoria en la moral. Con ello no quiero decir que no pueda poseer ese claro contorno, sino sólo que hoy en día no disponemos de ninguna fundamentación razonable a tal efecto.

Vuelvo a referirme ahora a aquello que caracterizaba como primer nivel de nuestra inestabilidad moral, a la cuestión de cómo la moral, que se había sustentado en todas las sociedades naturales sobre elementos religioso-tradicionales, se deja fundamentar de una manera natural. Esta cuestión acerca de una fundamentación natural de la moral tiene simplemente el sentido de interesarse por una fundamentación de la moral sin recurrir a cualquier tipo de verdades más elevadas, sean religiosas o metafísicas, las cuales no serían identificables de manera general e intersubjetiva.

En este planteamiento, parto del hecho de que el contenido a fundamentar es la consideración de los intereses de los otros. Podríamos enunciar asi la cuestión: ¿existe algo que nos motive de forma natural, desatendiendo nuestros propios intcreses, a tomar en consideración no sólo los intcreses de nuestros familiares y amigos, sino los intereses de todos los demás? - con lo cual dejo abierta por el momento la pregunta que formulaba anteriormente sobre quiénes son todos. Pues bien, se diría que, entre los modernos, cunden dos grandes corrientes que intentan mostrar cómo puede responderse positivamente a csa pregunta. Una re- 
curre al sentimiento altruista, como benevolencia o compasión. La teoría ética más importante que se ha fundamentado de esta manera es el utilitarismo. En nuestro contexto no se trata de hacer referencia a ciertas peculiaridades polémicas del utilitarismo. La tesis específica del utilitarismo sostiene que entre varias alternativas de acción hay que preferir siempre aquella que en conjunto comporta más felicidad o menos desdicha, sin importar cómo estén distribuidas ambas; cuando se va a conseguir una gran cantidad de felicidad, aunque unos pocos tengan que sufrir, hay que conformarse con las desventajas concomitantes. En esto reside la premisa de que la benevolencia o la compasión se aplica, por encima de los individuos, a una masa para la que la felicidad o el sufrimiento son intercambiables, a lo cual puede replicarse que el objeto de nuestra benevolencia o compasión lo son mucho más propiamente los individuos mismos. Pero estas disensiones no deben preocuparnos ahora. Con tal motivo, no hablo propiamente de utilitarismo, sino de una ética de corte utilitarista, a cuyo efecto dicho utilitarismo debe ser definido como el intento de fundamentar la ética en un sentimiento altruista, sin importar cómo se interpreta a continuación ese sentimiento. Ahora bien, toda la concepción choca con dificultades fundamentales: en primer lugar, existen virtudes morales que no son comprensibles partiendo de esas premisas, cuales son la confianza y la justicia; pero, sobre todo, en segundo lugar: si la moralidad fuese competencia del sentimiento, el cual poseen meramente de hecho diferentes hombres -unos más, otros menos-, tal y como algunos albergan el sentimiento contradictorio de experimentar placer en el sufrimiento de los demás, entonces la moral no sería algo que puede exigirse recíprocamente, ni el comportamiento moral sería algo a lo que estamos obligados. La teoría de los sentimientos no puede explicar el carácter de obligatoriedad de la moral.

La otra gran corriente que se ocupa de la fundamentación natural de la moral es el contractualismo. Micntras que el utilitarismo no puede hacer comprensibles de manera correcta las virtudes de la cooperación, el contractualismo se coloca exactamente en el lugar de la imprescindibilidad natural de la cooperación. Redunda en provecho de cada individuo el que todos estén sujetos a reglas de confianza y de consideración, y son estas reglas las que conceptuamos como morales. La naturalidad de esta aclaración de la moral se muestra tanto en la existencia universal de tales reglas en todas las sociedades, como en el hecho de que recurramos en la educación moral a ese tipo de racionalidad con fórmulas de este tenor: "desearías que los otros..., por consiguiente, etc.». Mientras que el utilitarismo descansa sobre un sentimiento altruista, el contractualismo se basa en el interés egoísta. Ahora bien, hay que cuestionarse hasta qué punto esa base egoísta puede soportar tanto lo que concierne a la motivación como a la obligación moral. Por ello, se ha objetado repetidas 
veces contra el contractualismo que de él no puede resultar ninguna moralidad real, sino únicamente un trueque. Las acciones morales no acontecen por mor de los demás, contrariamente a la concepción altruista; una moral semejante ino scría tan sólo una especie de sagacidad más refinada, comportándose como más astuto aquel que únicamente aparentase comportarse moralmente? En este sentido se expresó ya Platón en el libro II de La República.

No obstante, por lo que respecta a la obligatoriedad, el contractualismo llega ciertamente más lejos que el utilitarismo, aunque todavía no lo suficientemente lejos. Hemos visto que el utilitarismo no puede hacer comprensible en absoluto el carácter obligatorio de la moral. Pues bien, en el contractualismo se puede hablar ciertamente de compromiso, aunque se trate solamente del que la persona asume hipotéticamente en el caso de que el otro también actúe así, y sería incluso suficiente que la persona aparentara comprometerse. Sin embargo, la obligación moral no puede consistir ni mucho menos en el compromiso hipotético de una persona, sino que ha de ser válida objetivamente para ella, tanto si quiere como si no.

Estos déficit han dado lugar a diversos intentos de profundización en la postura contractualista por medio de supuestos adicionales más sólidos. Un intento semejante es el kantiano, según el cual, aquello que nos obliga a actuar moralmente es la razón. Por lo tanto, no se trataría sólo, como para el contractualismo, de pretender intereses particulares racionales que las reglas morales validaran socialmente, sino que sería mucho más razonable dejar de actuar moralmente guiados de forma relativa por el propio interés. Sin embargo, aquí parece que se hace un uso abusivo del concepto de razón. Apenas tiene sentido decir de una acción que es "mucho más" racional. Algunos filósofos contemporáneos piensan que la acción moral es sencillamente racional en el sentido de que está fundamentada en sí, pero también esto me parece ser una falacia. Una cosa sólo puede estar prácticamente fundamentada de forma rclativa de cara al establecimiento de fines; si se cree, por ejemplo, que una acción está fundamentada en sí misma, si puede fundamentarse frente a cualquiera en el sentido de que vaya en igual medida en interés de todos, entonces se ha establecido ya un criterio de moralidad con el equilibrio entre el interés de todos y con ello sólo se ha definido lo racional de forma relativa. La concepción kantiana de que la obligación interior, la cual relacionamos con la moral, sea la voz de la razón, se manifiesta, tal y como ya puso de relieve Schopenhauer, como una secularización de la voz de Dios.

Este intento de profundizar en el contractualismo no es, por consiguiente, un camino transitable, cuando uno se ha determinado a recurrir únicamente a fuentes naturales de la moral. Lo que necesitamos es una 
aclaración natural de la obligación moral, la cual se extienda más allá de la obligación meramente hipotética del contractualismo entendido en sentido estricto y que, sin embargo, no contraiga ningún empréstito trascendental, ni religioso ni metafísico. Tal explicación natural de aquello que se llama conciencia, la encontramos de forma rudimentaria en el psicoanálisis y en la psicología social. Freud intentó captar la configuración de la conciencia con sus conceptos del ideal del yo y del superyo. En su opinión, existe un núcleo de personalidad que vale al mismo tiempo para aquello que la persona misma quiere ser (ideal del yo) y lo que debe ser desde la perspectiva de los demás (superyo). Puesto que somos esencialmente seres sociales (también podemos decir con cautela: sólo si queremos ser seres esencialmente sociales), están conciliados en este núcleo de personalidad el querer y el deber de esta manera tan característica. Por deber se entiende: todos exigen de los demás reciprocidad en la observancia de las normas morales, y se exige esto en nombre de la identidad social constitutiva recíprocamente supuesta. Por esto denominamos a algo bueno o malo según se hayan seguido o infringido esas normas. De ahí que uno se comporte mal no bajo este o aquel respecto, sino como persona. En esto se basan también los sentimientos de la indignación, cuando otros actúan mal, y de la vergüenza y la culpa, si es uno mismo quien actúa mal. La fuente de esta obligatoriedad absoluta característica, en la que pensamos cuando hablamos de compromiso moral, no es, por consiguiente, ni religiosa ni identificable con la razón, sino que la constituye la colectividad; pero, opuestamente al contractualismo, sólo si el individuo entiende su propia identidad social. Esto no significa que la persona tenga que asumir las normas existentes en su entorno social fáctico; significa únicamente que pertenece a la esencia de las normas morales el que haya que entender socialmente el sentido de su carácter obligatorio. Tal y como fue subrayado por G.H. Mead, la conciencia del individuo puede dirigirse por completo contra las normas vigentes, pero cn ese caso con la pretensión de que aquellas normas, que quiere afirmar frente a las vigentes, estén constituidas de tal manera que puedan valer socialmente. Sin esta pretensión social la conciencia carecería del carácter de obligatoriedad.

Ahora bien, ¿cómo se puede cimentar la débil obligatoriedad del contractualismo por medio de esa fuerte obligatoriedad que reside en la conciencia? La conciencia parece ser, con sus implicaciones intersubjetivas, un indudable hecho antropológico, pero es en y por sí una mera estructura formal que puede ser rellenada a discreción por cualquier contenido normativo. Esta estructura es naturalmente constitutiva, en particular para todos los contenidos morales tradicionales religiosamente fundamentados; desde luego se diría, en primer lugar, que dicha estructura, a causa del concepto valorativo de identidad que presupone, sólo es 
realizable por una moralidad tradicional, ya que ésta implica una identidad semejante en lugar de una valorativa, mientras que el contractualismo ya no cuenta con dicha identidad valorativa. El yo está compuesto para el contractualismo únicamente por la suma de sus intereses egoístas, con lo que no puede encontrarse aquí un núcleo de personalidad tal, al que remita la conciencia, que sea comprendido socialmente.

Muchos autores no han prestado atención a estas dificultades y han alegado exclusivamente el hecho de que, como el contractualismo existe ya en y para si en el reconocimiento que hace cada uno del propio interés, he aquí que se mantiene con mayor razón si está apoyado por la conciencia. Sólo si cada uno introyecta las normas en su conciencia, puede conseguirse que éstas sean seguidas en general.

Este argumento es, dentro de sus limitaciones, evidente. El interés propio reconocido tiene que afirmar la formación de la conciencia. Sólo resta la cuestión de saber, en suma, si dispone por sí mismo de medios para formar una conciencia. Puesto que la conciencia implica un querer que excede al propio interés, no puede proceder de este último. Así nos encontramos ante la cuestión de cómo puede formarse en general una conciencia bajo condiciones naturales, ya no religiosas. A esto ha respondido G.H. Mead que la individualidad sólo emerge en el reconocimiento recíproco de las personas. La apreciada identidad que se presupone en la conciencia, habría ganado con ello un contenido natural. Queremos concebirnos reconociendo esencialmente a otras personas y, al mismo tiempo, siendo reconocidos por ellas y exigiendo esto de ellas. Pensamientos semejantes sobre la constitución de la individualidad en el reconocimiento recíproco se encuentran ya en Fichte y Hegel. También para Piaget se fundamenta la verdadera moral, que ya ha dejado de ser infantil, en el respeto mutuo. Al mismo tiempo, concibe esta moral como contractualista. Pero Piaget no ha explicado cómo están relacionados el contenido contractual y la forma del reconocimiento mutuo, ni ha aclarado qué hay que entender por respeto.

Por consiguiente, la cuestión queda por de pronto planteada de esta forma: ¿qué es, pues, lo que reconocen unas en otras las personas que se reconocen mutuamente? La respuesta de Fichte y Hegel era: su libertad, su autonomía. Pero es cuestionable si sobre esto puede ya fundamentarse una moral. Fichte lo ha creído así, Hegel no. En cambio, para Kant la autonomía era sólo el criterio para șaber cuándo un ser tiene un valor absoluto. Y lo que es reconocido es ese valor absoluto o, como Kant lo formuló también, el que la persona sea un fin en sí mismo. Pero ¿qué hay que representarse bajo un valor absoluto o un fin en sí? ¿No sería esto de nuevo algo metafísico? Podemos empero comprender a Kant e incluso el asunto mismo de la siguiente manera: no es que las personas sean ya en y por sí mismas fines en sí y entonces puedan también reco- 
nocerse mutuamente como tales, sino que en la medida en que se reconocen mutuamente se constituyen como fines en sí; y reconocer a alguien como fin en sí, como portador de un valor absoluto, significa no tratarlo como a un valor instrumental, y esto supone imponerse el precepto de no instrumentalizarle. Y prosiguiendo la explicitación, cabría decir que esto significa reconocerle como un sujeto de derechos. Por lo tanto, habría que entender el reconocerse mutuamente como otorgación recíproca de derechos.

Aquí tendríamos, pues, al mismo tiempo, una base para el concepto de justicia. Ni el utilitarismo ni el contractualismo conducen a un concepto semejante; el utilitarismo no lo hace así, porque su concepto del bien es acumulativo y no contiene ninguna norma de distribución; el contractualismo tampoco, porque sobre su base sólo puede acontecer que sean injustas aquellas acciones que contradigan el contrato, pero el contrato como tal no contiene por sí mismo ninguna cláusula referente a la justicia. En general, la justicia parece presuponer una pauta, y nosotros sólo hemos alcanzado una pauta semejante ahora, con la moral del reconocimiento mutuo como fin en sí. Por consiguiente, esta moral se muestra como mucho más irrenunciable, como si no pudiera pensarse bien una moral, en particular una moral política, sin el punto de vista de la justicia. Por ello, las morales tradicionales han contenido siempre un concepto de justicia, con lo cual, según aquello en lo que esas morales se apoyaran, el valor de la persona podía ser también desigual. Sin embargo, la justicia, sobre la base del reconocimiento como fin en sí, es esencialmente igualitaria, ya que no existe ninguna gradación dentro del concepto de fin en sí.

Ahora queda todavía por resolver otra cuestión: ¿en qué consisten los derechos mismos por lo que respecta al contenido? Podemos responder la pregunta afirmando que los contenidos de los que se trata son las normas que han resultado del contractualismo. El contractualismo habria encontrado ahora realmente un profundo apoyo, en la medida en que con esas normas ya no se trata de un trueque, sino que las personas pueden exigir el cumplimiento de esas normas como su derecho. Respetar ese derecho es ahora obligatorio para aquellos que se conciben como miembros de esta comunidad de derecho.

Pero aún podemos preguntarnos para qué necesitamos todavía el contractualismo como tal. El contractualismo es definido como aquello según lo cual la observancia general de las normas de la cooperación y de la toma de consideración descansa en los intereses egoístas de cada uno. Se mostraba después que el contractualismo necesita un apoyo, y que parece encontrarlo en la moral del respeto mutuo. El contractualismo no puede prescindir de una moral semejante, pero, si una vez poseemos una moral de este tipo, ésta será desde un principio más fuerte que 
el contractualismo y, por su parte, no dependerá de éste. La motivación contractual se vuelve superflua y puede, por consiguiente, tener a lo sumo el sentido de una intensificación adicional si las normas de la cooperación y de la toma de consideración se encuentran ya cimentadas en el respeto mutuo.

Sin embargo, entonces surge la pregunta de cómo la moral se ve motivada a su vez por el respeto mutuo. Solamente he ensayado la posibilidad, completando a Mead, de no poner de manifiesto la necesidad de una identidad social, que sería el fundamento requerido por una formación natural de la conciencia. Y la mera referencia a la realidad - el que la mayoría de los hombres tienen de hecho una conciencia- no puede ser suficiente, ya que puede interpretarse como un mero residuo de la tradición. La pregunta decisiva tiene que rezar así: ¿queremos tener una identidad social esencial, queremos concebirnos como miembros de una comunidad de sujetos de derecho que se reconocen mutuamente? ¿Existe para ello una motivación natural? La tesis de Mead, acerca de que sin una identidad semejante no se puede construir en absoluto una individualidad, parece ser demasiado fuerte. ¿Puede uno decir, por lo menos, que sin semejante reconocimiento recíproco de derechos ninguna persona puede tener un sentimiento de autovaloración, $o$, al menos, ningún sentimiento equilibrado de autovaloración? En todo caso, hasta el momento no han encontrado estas preguntas ninguna respuesta satisfactoria y, mientras no la encuentren, está en el aire la idea de una moral del respeto mutuo.

Si volvemos ahora la vista atrás, sobre los diferentes planteamientos modernos para conseguir una base natural para la moral, resulta que: tanto para una moral de corte utilitarista como para una de talante contractualista existe, sin duda, una motivación natural, a saber: en un caso el sentimiento natural de compasión; en el otro el del interés propio de hecho. Sin embargo, en el utilitarismo parece no subsistir ninguna posibilidad de encontrar algún compromiso en esta motivación intuitiva, cuando semejante obligación es constitutiva para la moral. El contractualismo, por su parte, contiene sólo una forma insuficiente de obligatoriedad. Por el contrario, la moral del respeto mutuo da una respuesta satisfactoria a la pregunta por la obligatoriedad, aunque, de otro lado, deja sin aclarar si existe una motivación natural autocomprensiva; y esto significa que queda abierta la cuestión de si tiene, en suma, una base natural, como los otros dos planteamientos, y no es únicamente un constructo.

Llegados a este punto, echemos finalmente una ojeada retrospectiva sobre esos problemas experimentados hoy en día de forma tan crítica, y que caractericé como los del segundo nivel; se diría entonces que justo aquella concepción incapaz de explicar en modo alguno la obligatorie- 
dad de la moral, esto es, el utilitarismo, es mucho más apropiada para hacer comprensibles las diferentes aplicaciones de la responsabilidad moral. Esto tiene su fundamento en que tanto el contractualismo como la moral del respeto mutuo se proponen esencialmente como objetivo la reciprocidad, y por consiguiente, las relaciones simétricas, mientras que el sentimiento moral es una relación asimétrica. La posición más limitada es la del contractualismo. Tropieza con el problema de los minusválidos, como, en general, con el de todos los perjudicados. Puesto que quien, por naturalcza o por cualquier otros motivos contingentes, ha sido privilegiado, puede no tener un interés egoísta en establecer con personas perjudicadas compromisos que obligan a todos por igual. Esto es de otra manera en una moral que persigue una reciprocidad del respeto mutuo ideal, no material; esta moral ordena que todas las personas se respeten por igual, y de esto resultan obligaciones incluso más fuertes para con los minusválidos. Por lo que respecta a las generaciones futuras, el contractualismo fracasa por completo, pero también llega a su límite la moral del respeto mutuo. Los que van a vivir después son, sin duda, también personas, pero la responsabilidad, si es que aún existe, ya no descansa en una reciprocidad. Sin embargo, esta dificultad se deja remontar en la medida en que validemos un diálogo moral contrafáctico, ficticio, lo mismo que cuando nos referimos a una promesa hecha a alguien que ya ha muerto. Ahora bien, tampoco es posible de cara a fetos, niños pequeños y animales. Estos no son personas, no pertenecen a la comunidad moral, si ésta queda constituida por el reconocimiento mutuo. Por el contrario, el utilitarismo no tiene ninguna dificultad en hacer comprensible la toma en consideración de fetos, niños pequeños y animales. Como ya dijo Bentham: "La pregunta no es si pueden pensar o si pueden hablar, sino si pueden sufrir». Pero el utilitarismo, por su parte, se ve incapaz de explicar, tanto en este punto como en cualquier otro, que nos hallamos obligados para con esa responsabilidad en aras de tal consideración. El fracaso de la filosofía moral moderna se muestra aquí en su forma más drástica. La responsabilidad frente a los niños parece el caso intuitivamente más sencillo de una obligación moral, y, sin embargo, no tenemos ninguna teoría moral que pueda explicarla. 